\title{
L'utilisation du béton pour les ouvrages maritimes : synthèse franco-anglaise des bonnes pratiques
}

\author{
S. DUPRAY ${ }^{1,2}$, G. ROBERTSHAW ${ }^{3}$, N. BEAN ${ }^{4}$, K. GAMST $^{4}$
}

${ }^{1}$ CETMEF, 2 Boulevard Gambetta BP 60039, 60321 - COMPIEGNE Cedex

sebastien.dupray@developpement-durable.gouv.fr

${ }^{2} \mathrm{HR}$ Wallingford, WALLINGFORD, Oxfordshire, OX10 8BA, GB

s.dupray@hrwallingford.co.uk

${ }^{3}$ Halcrow, Deanway Tech. Centre, HANDFORTH, Cheshire, SK9 3FB, GB

robertshawg@halcrow.com

${ }^{4}$ CIRIA, Classic House, 174 - 180 Old Street, LONDON EC1V 9BP, GB

\section{Résumé :}

\section{kristina.gamst@ciria.org}

L'article présente une recherche Franco-anglaise en cours sur l'utilisation du béton pour les ouvrages maritimes et quelques résultats intermédiaires.

Le projet s'intéresse avec différents niveaux de détail aux enjeux, aux spécificités de différentes familles d'ouvrages, aux agents extérieurs et autres phénomènes à considérer, au matériau béton, au dimensionnement - en particulier pour la durabilité -, au contrôle qualité et aux essais spécifiques, à la construction et à l'entretien / réparation des ouvrages existants.

Un guide des bonnes pratiques devrait être disponible fin 2009 ou début 2010 en Français après traduction de document de travail en langue Anglaise.

\section{Abstract :}

The present paper presents an on-going English-French research on the use of concrete for maritime structures and the key intermediate findings.

The project focuses - with different levels of detail - on the key drivers, on the specificities for various structure families, on external agents and other phenomenon to consider, on the concrete material, on design - in particular for durability -, on quality control and testing, on construction, and on maintenance and repair of existing structures.

A good practice guidance document should be available by the end of 2009 or early 2010 in French after translation of the research report.

\section{Mots-clés :}

Ouvrages côtiers - Ouvrages maritimes - Béton - Durabilité - Construction Entretien / Maintenance 


\section{$1 \quad$ Introduction}

\subsection{Motivations de l'équipe projet}

Les ouvrages en béton forment une part importante des ouvrages neufs ou existant du parc national d'ouvrages maritimes, portuaires ou côtiers. En effet, le béton a été très largement utilisé pour la construction, la réparation et la reconstruction des ouvrages dans le domaine maritime et côtier et continue de l'être. On peut estimer que $20 \%$ des ouvrages de lutte contre la mer ou des ouvrages maritimes est composé de béton sous ses différentes formes, à savoir coulé en place ou comme éléments préfabriqués voire une combinaison des deux. Le béton est par ailleurs aussi largement utilisé pour d'autres types d'ouvrages comme les ouvrages portuaires tels que les quais, les écluses, les formes et certaines superstructures (qui ne sont pas spécifiquement abordés dans le projet).

La documentation méthodologique sur le sujet des ouvrages maritimes en béton est fragmentaire et il n'existe actuellement aucun guide autoporteur présentant les bonnes pratiques couvrant la conception et le dimensionnement, les matériaux, la construction, l'entretien et la réparation de ces ouvrages. Il est à noter que la situation est la même en Angleterre où, là aussi, il existe un corpus de recommandations et de guides particuliers sur des sujets spécifiques comme par exemple la stabilité ou la performance hydraulique, les matériaux, la durabilité etc.

De plus, le changement - récent ou en cours - du référentiel normatif ou réglementaire requiert une remise à plat des approches antérieures ainsi qu'une mise à jour des références à considérer.

\subsection{Particularités du béton maritime}

Même si les phénomènes en jeu dans l'environnement maritime peuvent aussi être rencontrés à terre, l'intensité et la combinaison de ces phénomènes ainsi que la nature des ouvrages maritimes et côtiers rendent l'utilisation du béton pour les ouvrages maritimes tout à fait particulière. Parmi les challenges associés à l'utilisation du béton pour les ouvrages maritimes et côtiers il faut notamment citer la durabilité du béton pour la durée de vie de l'ouvrage, en particulier :

- le renforcement des bétons en minimisant ou s'affranchissant de la corrosion des renforcements en acier,

- la lutte contre la pénétration des chlorures,

- la lutte contre l'attrition des bétons,

- l'utilisation des fibres et autres matériaux non corrodables,

- les nouvelles approches européennes pour spécifier les bétons, leurs composants en lien avec le dimensionnement pour la durabilité et la résistance aux charges hydrauliques et structurelles. 
L'utilisation du béton pour la construction est aussi un challenge particulier associé soit aux conditions environnementales spécifiques soit aux particularités liées à certains ouvrages, notamment :

- le bétonnage sous eau ou dans la zone de marnage,

- la préfabrication d'éléments de construction ou blocs artificiels qui requièrent généralement un décoffrage rapide ou des techniques de coffrage particulières,

- le recours à des éléments massifs sujets aux problèmes thermiques lors de l'hydratation du béton,

- l'exposition du béton au jeune âge à l'action de l'eau de mer ou des vagues.

\subsection{Objectif du projet}

L'ambition du CETMEF, en s'associant au CIRIA Britannique, est de produire un guide des bonnes pratiques, de rassembler les expériences sur les ouvrages maritimes et côtiers en béton. Les derniers changements normatifs au niveau européen contribuent au besoin de recommandations structurées mais le travail en cours n'a ni vocation à reproduire la documentation européenne existante, par ailleurs disponible sous d'autres formes, ni d'être un travail normatif. Il a pour vocation de mettre en commun les expériences spécifiques des différents partenaires, d'être un lieu d'échange entre les maîtres d'œuvre, les entreprises de travaux et les spécialistes du matériau béton notamment.

\section{Contenu et organisation du projet}

\subsection{Lectorat}

Le guide s'adresse à des lecteurs ayant un minimum de connaissance en génie civil et dans le domaine maritime en particulier. Le lecteur (voir liste ci-dessous) pourra y trouver des informations plus ou moins appropriées à son usage $(+++$ : très appropriées ; + peu appropriées) :

- Maîtres d'ouvrage : ++.

- Maîtres d'œuvre : +++.

- Projeteurs et bureaux d'études spécialisés : +++ .

- Industriels du béton : +++.

- Entreprises de travaux maritimes : +++ .

- Laboratoires d'essais : ++.

- Universitaires et enseignants : +. 


\subsection{Ouvrages considérés}

Afin de proposer une approche rationnelle de l'utilisation du béton pour les ouvrages maritimes et côtiers, les ouvrages ont été regroupés en familles d'ouvrages présentant des similarités lors du dimensionnement, de la construction et de la gestion du cycle de vie. Les ouvrages sont ainsi rassemblés en 9 familles, à savoir les murs, les ouvrages côtiers, les ouvrages en éléments préfabriqués, les blocs artificiels pour les digues, les revêtements, les caissons, les ouvrages portuaires exposés, les tours, les ouvrages composites, voir Figure 1.

\subsection{Travaux antérieurs}

L'utilisation du béton pour les ouvrages maritimes n'a fait que peu l'objet de publications autoporteuses. Néanmoins, les documents ci-dessous forment une partie du corpus technique en la matière et le présent projet s'appuiera notamment sur ces documents :

- le Guide sur l'utilisation du béton en sites aquatiques (THAUVIN\&MENGUY, 2008) s'intéresse particulièrement au matériau béton et à sa durabilité.

- Les bétons et les ouvrages en site maritime (CIMBETON, 2008) traite spécifiquement du matériau béton et aborde le béton armé.

- le Guide sur l'utilisation des enrochements pour les ouvrages hydrauliques (CIRIA / CUR / CETMEF, 2007) présente les blocs artificiels en béton et leur dimensionnement hydraulique (à paraitre en français).

- Concrete in coastal structures (ALLEN, 1998) regroupe divers articles sur le sujet (en anglais).

- Evaluation and repair of concrete structures (USACE, 1995) (en anglais).

- Underwater concrete (FRANKLIN et al.,1990) traite en particulier de la mise en œuvre (en anglais).

- Stresses in concrete armour units (DAVIDSON\&MAGOON, 1990) traite des contraintes dans les blocs artificiels et les essais sur prototypes (en anglais).

- Concrete in marine environment (MALHOTRA, 1988) (en anglais).

- Maritime concrete (CONCRETE SOCIETY, 1986) est un compte rendu d'un symposium sur le béton pour les ouvrages maritimes.

- Innovative design of concrete armour units (CUR, 1986) est une synthèse sur la résistance des blocs artificiels en béton (en anglais).

Certains environnements maritimes particuliers font aussi l'objet de publications spécifiques (WALKER, 2002), (TOUTLEMONDE et al., 2007). 


\subsection{Organisation du contenu}

Le guide s'articule en huit chapitres techniques (voir Figure 2), plus un chapitre introductif, à savoir :

- Le Chapitre 2 présente les enjeux associés à l'utilisation du béton pour les ouvrages maritimes, en particulier les exigences sur la durée de vie de service, l'optimisation de la solution technique, la constructibilité, les risques et, l'hygiène et la sécurité, les aspects environnementaux, architecturaux et les coûts.

- Le Chapitre 3 présente un aide mémoire pour chaque famille d'ouvrages qui synthétise les particularités à considérer lors de la conception et du dimensionnement, de la construction et de l'entretien et la maintenance.

- Le Chapitre 4 présente les agents environnementaux et les autres sources de dégradations potentielles à considérer.

- Le Chapitre 5 traite du béton, en particulier des constituants, de la formulation (y compris la qualification au laboratoire et in situ) et de la fabrication du béton.

- Le Chapitre 6 s'intéresse au dimensionnement en particulier pour la durabilité sur la durée de vie de service pour résister aux agents environnementaux et dans le cas d'ouvrages spécifiques.

- Le Chapitre 7 aborde le contrôle qualité et les essais particuliers à l'environnement maritime pour le béton ou les éléments en béton.

- Le Chapitre 8 traite de la construction, en particulier sous l'eau, dans la zone de marnage.

- Le Chapitre 9 aborde l'entretien et la réparation des ouvrages maritimes en béton. 
Ouvrages portuaires et gestion des sédiments
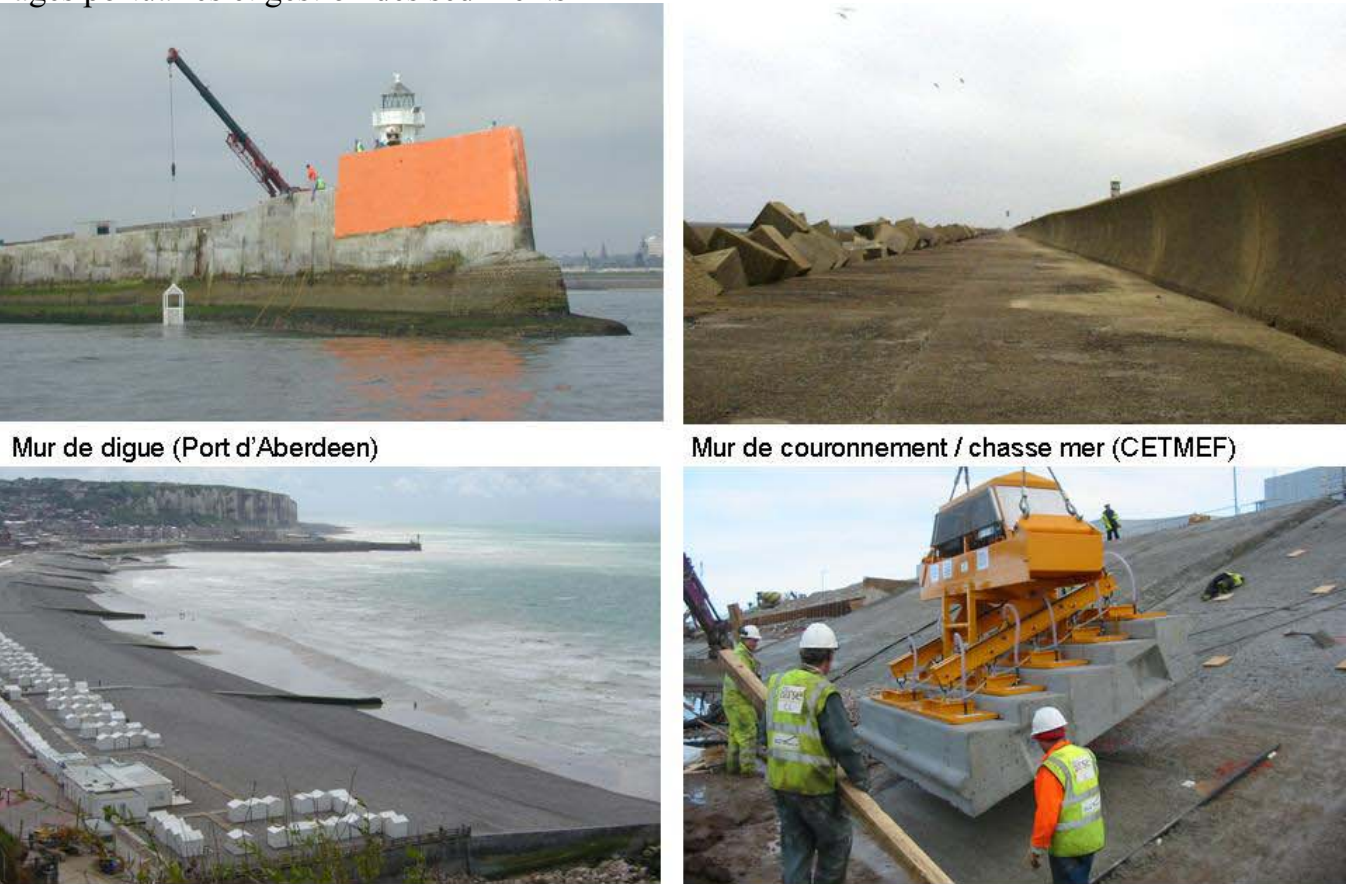

Mur de couronnement / chasse mer (CETMEF)

Epis béton (DDE80)

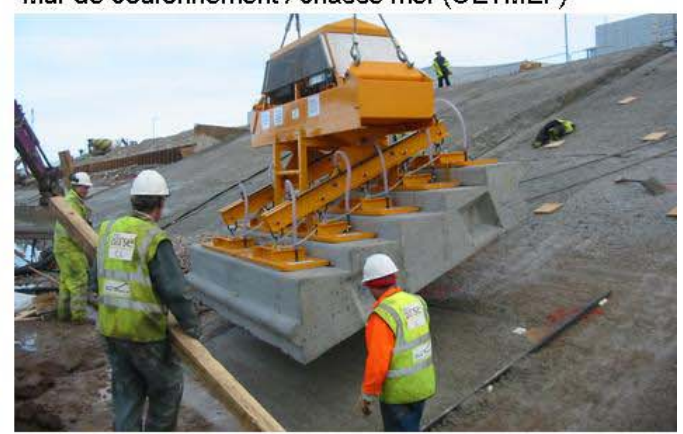

Revêtement de protection côtier (Birse)
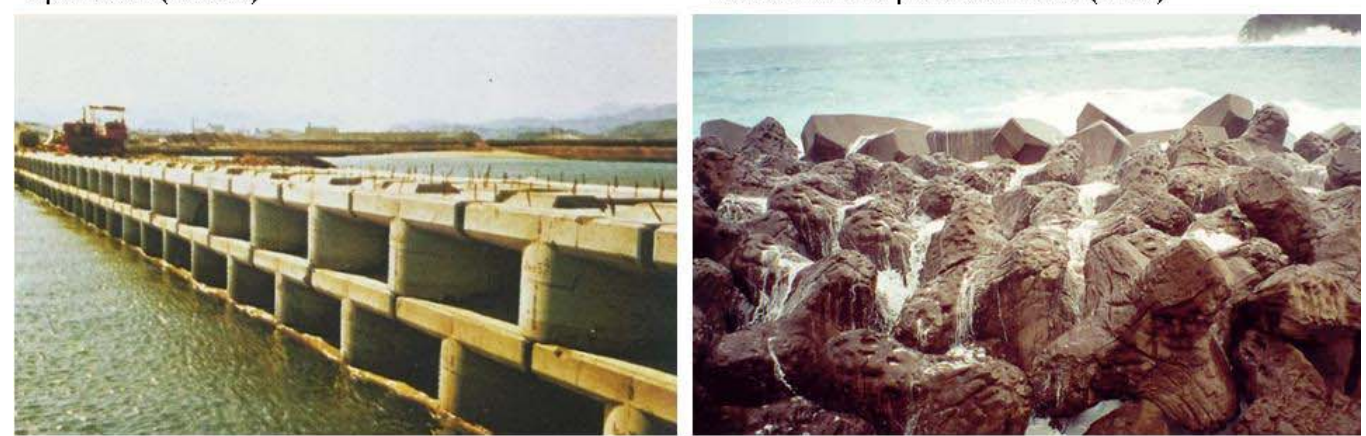

Eléménts préfabriqués (CLI)

Bloc artificiels pour digues (CLI)

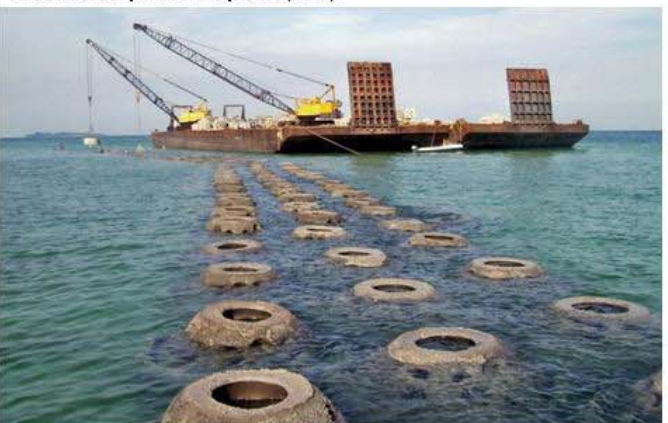

Elément pour récifs artificiels ( $\mathrm{L}$ Harris)
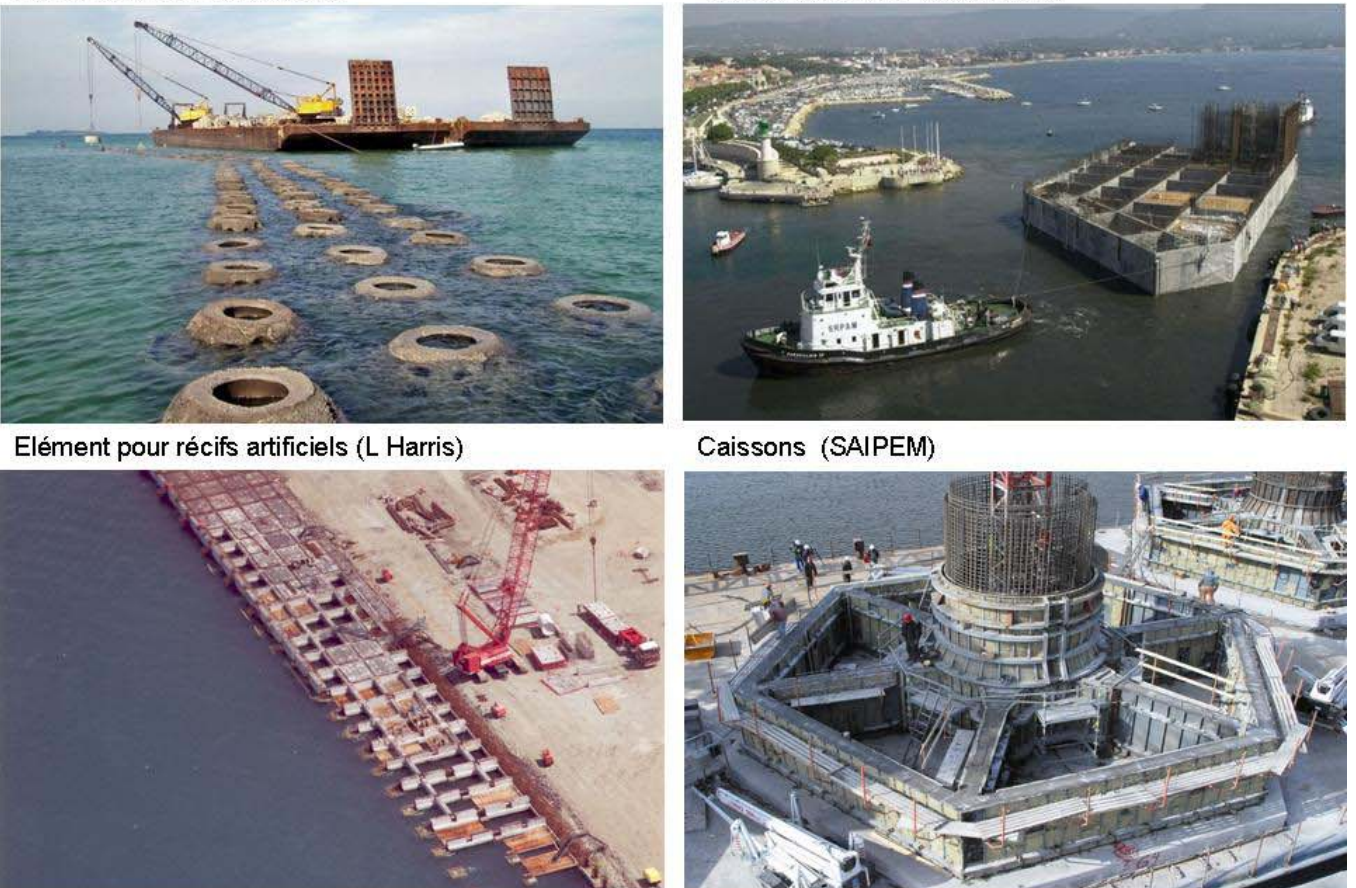

Caissons (SAIPEM)

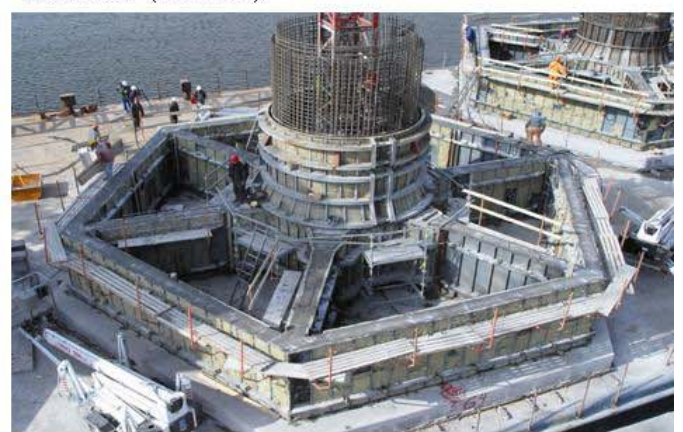

Element préfa comme coffrage permanent (SAIPEM)

Tour éolienne (Concrete Center)

Figure 1. Illustration des ouvrages couverts par le guide 
Ch 3. Famille d'ouvrages et particularités à considérer lors de

- la conception et du dimensionnement,

- la construction,

- l'entretien et la maintenance.

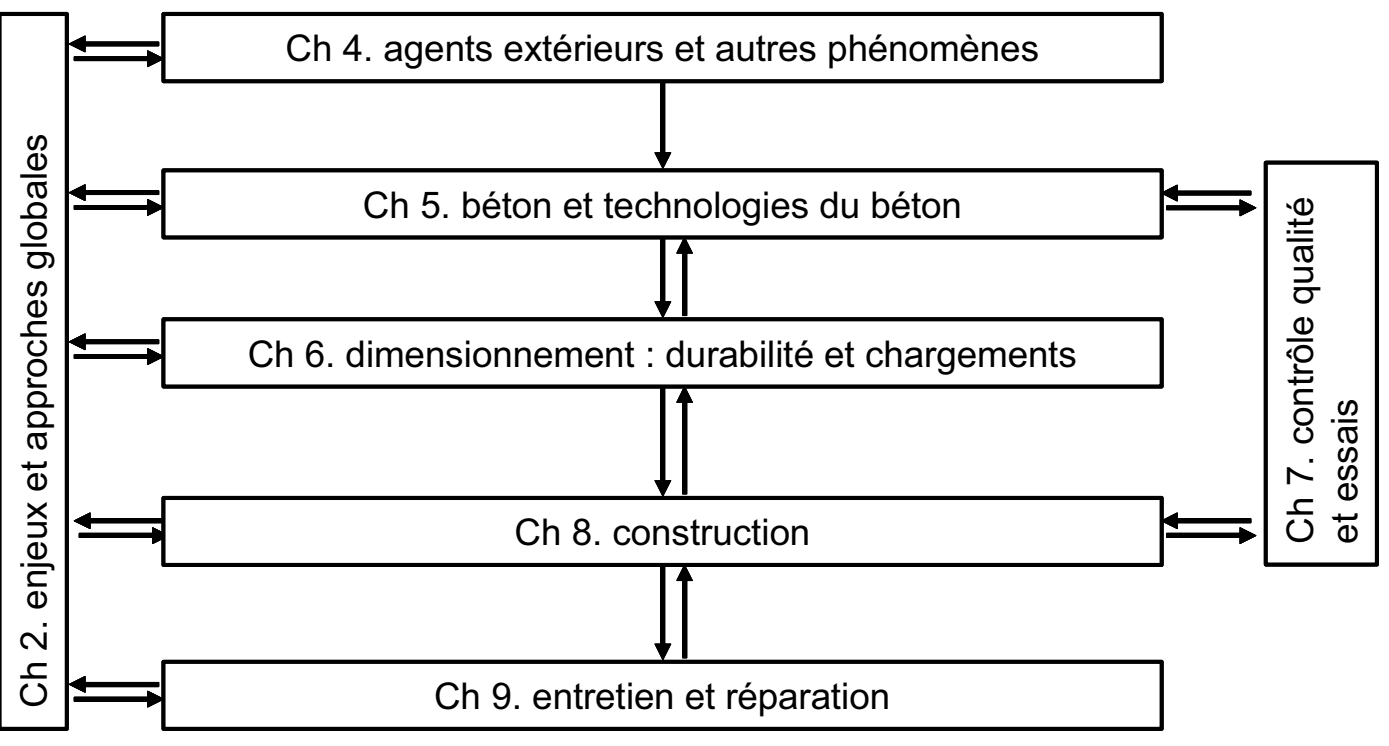

Figure 2. Logigramme d'organisation des chapitres du guide

\section{$3 \quad$ Résultats partiels}

Les travaux du groupe de travail sont en cours. Néanmoins, les sujets suivants apparaissent comme des enjeux majeurs qui seront détaillés.

\subsection{Durabilité sur la durée de vie de l'ouvrage}

Le dimensionnement de l'ouvrage pour la durabilité et la résistance mécanique aux sollicitations sont particulièrement importants pour le béton dans les ouvrages maritimes. Pour ce qui concerne le dimensionnement structurel, la prise en compte des sollicitations dynamiques ou de fatigue induites par les impacts de la houle est la principale difficulté. La durabilité du béton est particulièrement importante dans l'environnement maritime. D'une façon générale, le béton doit être compatible avec la chimie de l'environnement marin, e.g. le ciment doit résister aux sulfates, et avec les dégradations particulières liées à l'action de la houle et des sédiments, e.g. la résistance à l'usure et aux impacts. Lorsqu'il est armé, la corrosion liée aux chlorures (puis à la carbonatation) est critique. Par ailleurs, le caractère massif des éléments / structures maritimes induit des problèmes liés à la chaleur d'hydratation du béton, soit directement par la fissuration thermique, soit indirectement par le risque de réaction sulfatique interne. 
Le choix de la durée de vie de service de l'ouvrage est pour cela critique car celle-ci contrôle de fait les sollicitations à prendre en compte ainsi que la durabilité à atteindre. Par ailleurs, la conception de l'ouvrage, le choix des constituants, le dimensionnement du béton et la prise en compte des méthodes constructives doivent permettre d'atteindre la qualité requise pour la durée de vie de l'ouvrage.

Les approches normalisées (voir la norme EN206) ou recommandées (voir par exemple AFGC (2004) ou LCPC(2007)) devraient permettent de traiter le dimensionnement pour la durabilité, en particulier par l'utilisation de classes standards d'exposition ou d'approches performantielles. Néanmoins, il doit être souligné que l'emploi de ces méthodes peut s'avérer délicat dans le cas de durées de vie extrêmes pour lesquelles la détermination des paramètres et l'emploi des modèles sont délicats et requièrent des investigations parfois longues qui devraient être prises en compte lors de l'établissement du programme. Par ailleurs la compatibilité entre les exigences structurelles et de durabilité doit être assurée ce qui est parfois difficile. Des constituants ou renforcements particuliers, la protection cathodique, des traitements de surface permettent parfois d'atteindre ces niveaux d'exigences même si leur efficacité sur le long terme doit toujours être étudiée.

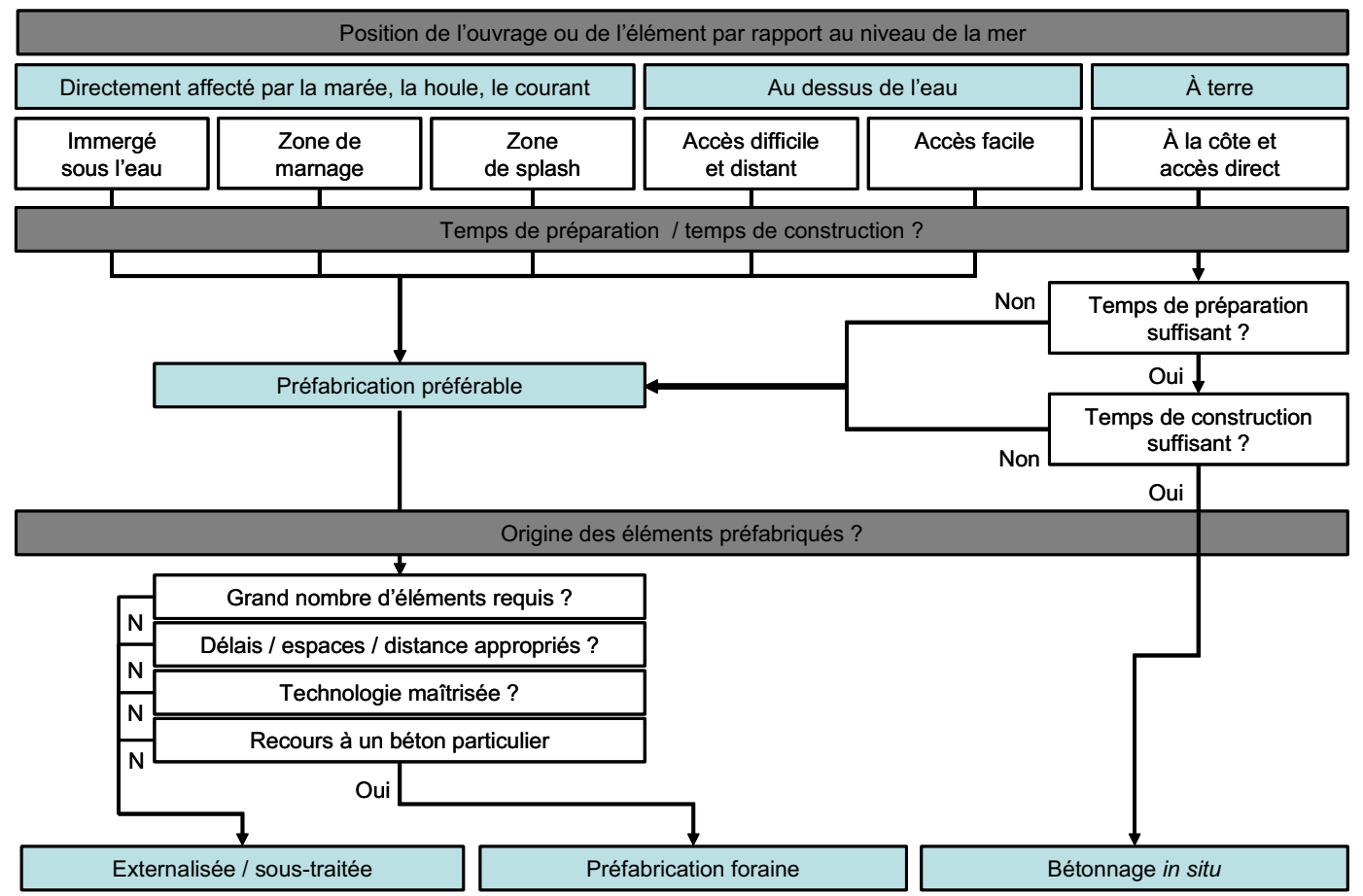

Figure 3. Logigramme de synthèse de l'emploi du béton in situ ou du recours à la préfabrication pour les ouvrages maritimes 


\subsection{Constructibilité comme pré-requis}

Par opposition à d'autres emplois du béton en génie civil, la constructibilité est critique pour les ouvrages maritimes. En effet, les conditions d'accès à la zone de construction, l'exposition à l'eau de mer et à l'action de la marée, de la houle et $\mathrm{du}$ courant, rendent certaines taches particulièrement difficiles, voire impossible. Par ailleurs, la construction doit pouvoir être réalisée dans des conditions convenables permettant in fine une mise en œuvre appropriée compatible avec la durabilité de l'ouvrage et du béton telle qu'étudiée au laboratoire.

Les deux situations extrêmes, à savoir le recours au bétonnage in situ et l'utilisation de la préfabrication sont présentés à la Figure 3. Le recours à des solutions composites, i.e. intégrant des éléments préfabriqués comme coffrage permanent du béton coulé en place, peut s'avérer une option intéressante.

\section{$4 \quad$ Implémentation}

\subsection{Equipe projet}

Une collaboration bi-partite sous l'égide du CIRIA en Angleterre et du CETMEF comme tête de réseau Français est en cours pour traiter les sujets abordés au 2.4 pour les ouvrages présentés au 2.2. Le CIRIA s'appuie notamment sur les équipes de HR Wallingford, de Halcrow, et de la Concrete Society pour mener à bien sa part du projet. De son coté, le CETMEF s'appuie sur différents professionnels du domaine, en particulier le Réseau Scientifique et Technique de l'Equipement, les Ports du Havre, de Marseille et de Nantes; des organismes professionnels comme le TRAMAF, le STRRES ou le CEFRACOR; des entreprises comme Eiffage ou Bouygues; des bureaux d'ingénierie comme la CNR, SAIPEM, CLI pour contribuer à sa part du projet

\subsection{Planning provisionnel}

Le planning provisionnel est le suivant :

- Cadrage et équipe projet - novembre 2007.

- Premier jet - février 2008.

- Second jet - fin juin 2008.

- Document final - fin 2008.

- Valorisation - début 2009.

- Traduction en français et production - courant 2009.

\section{$5 \quad$ Conclusion}

Le CETMEF élabore un guide autoporteur sur l'utilisation particulière du béton dans les ouvrages maritimes, i.e. la conception, les technologies des bétons, le dimensionnement, la construction et l'entretien maintenance, en partenariat avec 
le CIRIA (GB). Ce guide synthétise les bonnes pratiques, intègre les changements normatifs et réglementaires, et propose des pistes d'innovations.

Les résultats du projet devraient être disponibles fin 2008 en Anglais puis en 2009/2010 en Français après traduction.

\section{$6 \quad$ Références bibliographiques}

1. AFGC (2004). Conception des bétons pour une durée de vie des ouvrages. AFGC, Paris, 245p

2. ALLEN R. (1998). Concrete in coastal structures. Thomas Telford, London (GB), 301p

3. CIMBETON (2008). Les bétons et les ouvrages en site maritimes - Guide technique. CIMBETON, Paris, 45p

4. CIRIA / CUR / CETMEF (2007). Guide sur l'utilisation des enrochements pour les ouvrages hydrauliques - version français à paraître. CETMEF, Compiègne, $1268 \mathrm{p}$

5. CONCRETE SOCIETY (1986). Marine concrete - papers of the Int. Conf. on Concrete in the marine environment, London, 22-24 September 1986. Concrete Society, London (GB), 446p

6. CUR (1986). Breakwaters : strength of concrete armour units - Executive summary. CUR, Gouda (NL), 34p

7. DAVIDSONN D.D. \& MAGOON O. (1990). Stresses in concrete armour units. ASCE Publishing, New York (USA), 421p

8. FRANKLIN A. J. D. et al. (1990). Underwater concreting - Technical Report. Concrete Society, London (GB), 40p

9. LCPC (2007). Recommandations pour la prévention des désordres dus à la réaction sulfatique interne - Collection Guide technique. LCPC, Paris, 60p

10. MALHOTRA V. M. (1988). Concrete in the maritime environment - papers of the $2^{\text {nd }}$ Int. Conf. of St Andrews by-the-sea. A.C.I. , Detroit (USA), 737p

11. THAUVIN B. \& MENGUY M. (2008). Guide sur utilisation du béton en sites aquatiques. CETMEF, Compiègne, $53 \mathrm{p}$

12. TOUTLEMONDE F. (2007). Concrete under severe conditions - environment and loading - Actes de la $5^{\text {ème }}$ conférence internationale sur les structures en béton sous conditions extrêmes d'environnement et de chargement. LCPC, Paris, $1866 \mathrm{p}$

13. USACE (1995). Evaluation and repair of concrete structures - Engineer Manual 1110-2-2002. USACE, Washington DC (USA), 185p

14. WALKER M. (2002). Guide to the construction of reinforced concrete in the Arabian Peninsula - Publication C577. CIRIA, London (GB), 214p 